\title{
Large minimally symptomatic iatrogenic urinoma presenting three years after abdominal aortic aneurysm repair
}

\author{
Mark Spurrell, B. Eng; '* John Pike, MD, FRCSC; Gavin Duffy, MD, FRCSC ${ }^{\dagger}$ \\ *Memorial University of Newfoundland, St. John's, NL; 'Eastern Health, Newfoundland, Health Sciences Centre, St. John's, NL
}

Cite as: Can Urol Assoc J 2012;6(2):e50-53. htrp://dx.doi.org/10.5489/cuaj.10154

\begin{abstract}
We describe a case of a large, minimally symptomatic, urinoma presenting 3 years after emergent repair of a ruptured abdominal aortic aneurysm. We discuss the symptoms and signs, as well as the imaging, treatment and prevention options, for this rare complication. Because of the high mortality associated with delayed diagnosis of ureteral injury, a high index of suspicion should be maintained in patients who had surgery adjacent to the ureters.
\end{abstract}

\section{Case summary}

A 58-year-old man was undergoing investigations for thyrotoxicosis. The patient's surgical history included a technically difficult emergent repair of a ruptured abdominal aortic aneurysm (AAA) 3 years prior, as well as left leg amputation during the same admission. During the appointment, the patient complained of a 3-year history of vague feelings of "fullness" under his surgical scar in the left lower quadrant. A mass was palpated in the area on physical exam. On previous examination, a diagnosis of an incisional hernia was made. However, during this appointment, the examining physician questioned whether the mass was consistent with a hernia. Abdominal ultrasound was arranged.

The ultrasound of the mass revealed an anechoic fluid collection measuring a maximum of $11.8 \mathrm{~cm} \times 13.2 \mathrm{~cm}$ in the transverse plane and $21.1 \mathrm{~cm}$ in the coronal plane. Left hydronephrosis was also noted. Ultrasound guided drainage of the mass revealed a clear, tea-coloured fluid with inflammatory cells and pigment laden macrophages, but no bacteria or malignant cells. The post drainage image showed significant decompression. Repeat ultrasound of the area a week later revealed reoccurrence of a $12 \mathrm{~cm} \times 13 \mathrm{~cm} \times 10 \mathrm{~cm}$ mass, as well as marked pelvicaliectasis of the left kidney. The ureter could not be followed distally and was felt to be compressed by the mass. A computed tomography (CT) scan of the abdomen without a pyelographic phase was ordered by the original physician and the patient consulted a urologist as an outpatient. The CT scan showed a very thin parenchyma of the left kidney and ureteric obstruction (Fig. 1, Fig. 2). The scan was not repeated with a pyelographic phase as the chance of significant renal function in the left kidney was felt to be low.

Cystoscopy and retrograde pyelogram were undertaken to delineate the anatomy and assess for ureteric injury. Visualization of the bladder was normal. The ureter was not visualized beyond the distal third during attempted retrograde pyelogram. A guidewire could not be passed beyond this point.

An antegrade nephrostogram was performed for further assessment (Fig. 3). This showed significant leakage into the mass at about $2 \mathrm{~cm}$ below the ureteropelvic junction. This was consistent with a urinoma secondary to left ureteral dismemberment, which was felt to have occurred at the time of the patients previous AAA repair. Review of the operative record from the emergent ruptured AAA repair revealed a technically difficult surgery with multiple aortic clampings and a surgical field frequently obscured by bleeding.

Due to the age of the injury, as well as the extremely diminished parenchyma of the kidney, a left nephrectomy along with excision of the urinoma sac was carried out using an open approach. Laparoscopic nephrectomy was not an option due to extensive scar tissue from the past AAA repair. Initially, an adrenal sparing procedure was planned. However, intra-operatively, the adrenal was noted to be extensively adhered to the kidney due to the inflammatory pathology. A plane outside of the adrenal was noted as the easiest to follow, and therefore was the safest choice. Despite caution during the development of the plane, a small hole was inadvertently made in the diaphragm. It was repaired without difficulty. The urinoma mass, involved ureter, left kidney and left adrenal were removed as one intact mass (Fig. 4). Postoperative course was complicated 


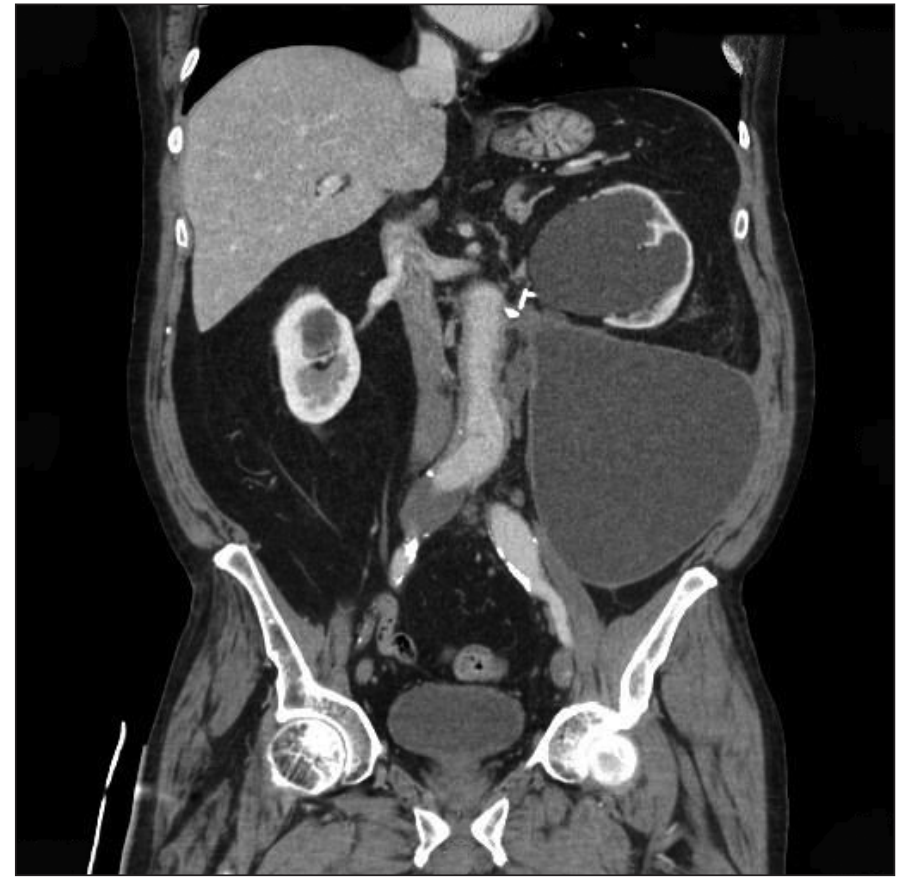

Fig. 1. Coronal computed tomography image of the abdominal urinoma. Extensive hydronephrosis of the left kidney is seen.

by broncospasm, congestive heart failure and pulmonary embolism. The patient was discharged on postoperative day 17. Pathological evaluation of the specimen revealed no signs of malignancy. When seen for follow-up 6 weeks later, the patient stated that he was feeling better than he had in many years. No further urological issues were noted. No recurrences have been noted in the past 14 months.

\section{Discussion}

Although rare, ureteral injury is a known complication of vascular reconstructive surgery. Injury is identified intraoperatively in $0.8 \%$ of these surgeries and arises as a late complication in $2.2 \%{ }^{1}$ Direct injury occurs in $0.67 \%$ to $0.85 \%$ of cases. Vascular surgery accounts for up to $8 \%$ of all iatrogenic ureteral injuries in both sexes, but $43.8 \%$ in males. $^{2}$

Injuries can be classified as direct or extrinsic compression. Direct injuries generally occur due to failure to recognize the ureter during dissection at the pelvic brim or poor use of clamps or suture ligation to control bleeding. Extrinsic compression tends to arise from poor tunnelling of a limb of the bifurcated arterial graft or as a result of retroperitoneal fibrosis. ${ }^{1}$ Risk factors for ureteral injury include peri-aneurysmal fibrosis associated with inflammatory AAA, pseudo-aneurysms, re-operative vascular reconstructions and extensive haemorrhage and fibrosis. ${ }^{3}$ Location of intraoperative injury tends to be the mid or distal portion of the ureter. ${ }^{4}$

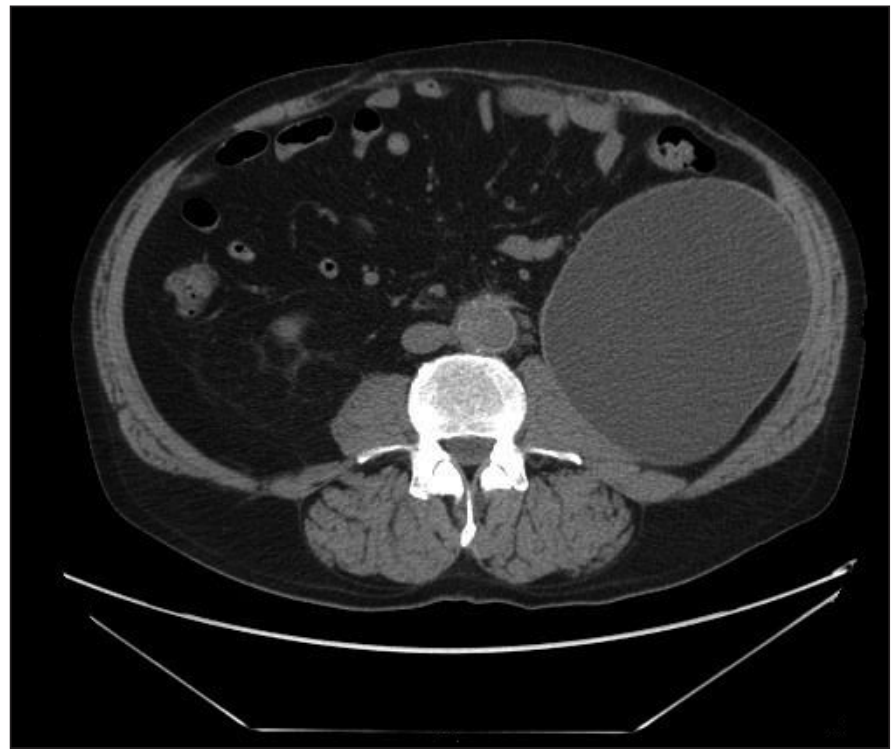

Fig. 2. Transverse computed tomography image of the abdominal urinoma.

An important contributing factor for morbidity associated with ureteral injury is delay in diagnosis. ${ }^{5}$ Of all iatrogenic ureteral injuries, $50 \%$ to $70 \%$ are not recognized acutely. ${ }^{4}$ Up to a $50 \%$ mortality is associated with delayed diagnosis. ${ }^{3}$ Unilateral flank pain is the most common presenting symptom. Fever, adynamic ileus or clear fluid drainage from incision or drainage sites may also occur. ${ }^{2}$ Additionally, gross hematuria or a contained fluid collection may indicate a ureteral injury. ${ }^{6}$ Half of patients are seen to have external urine drainage or a contained collection. ${ }^{6}$ However, up to a third of patients may be asymptomatic, as inert urine does not create an inflammatory reaction. ${ }^{2,3}$ Because of this, a high degree of suspicion for ureteral injury should be maintained in patients who had surgery adjacent to the ureters.

When ureteral injury is suspected, the most sensitive imaging study is a retrograde pyelogram. ${ }^{4} \mathrm{~A}$ contrast enhanced CT scan of the abdomen and pelvis is also a reasonable option and less invasive. If a fluid collection is identified adjacent to the ureter on the $\mathrm{CT}$, delayed images should be performed. ${ }^{3}$ Extravasation of the contrast is diagnostic for ureteral injury and lack of extravasation suggests abcess or hematoma. ${ }^{3}$ Intravenous urogram has been suggested as an option; ${ }^{1,7}$ however, the range of false negatives is $44 \%$ to $73 \%$, which is higher than the previously mentioned options. ${ }^{3}$ Ureteral injuries are classified by the American Association for the Surgery of Trauma (AAST) and can be used to guide management (Table 1). ${ }^{8}$

Ureteral transection occurring at the time of aortic reconstruction should be repaired immediately. Repair procedures will depend on location of the injury, as well as surgeon preference. Special attention should be paid to ensuring a watertight repair, as urinoma formation leads to increased risk of graft infection. An internal double J stent should be 


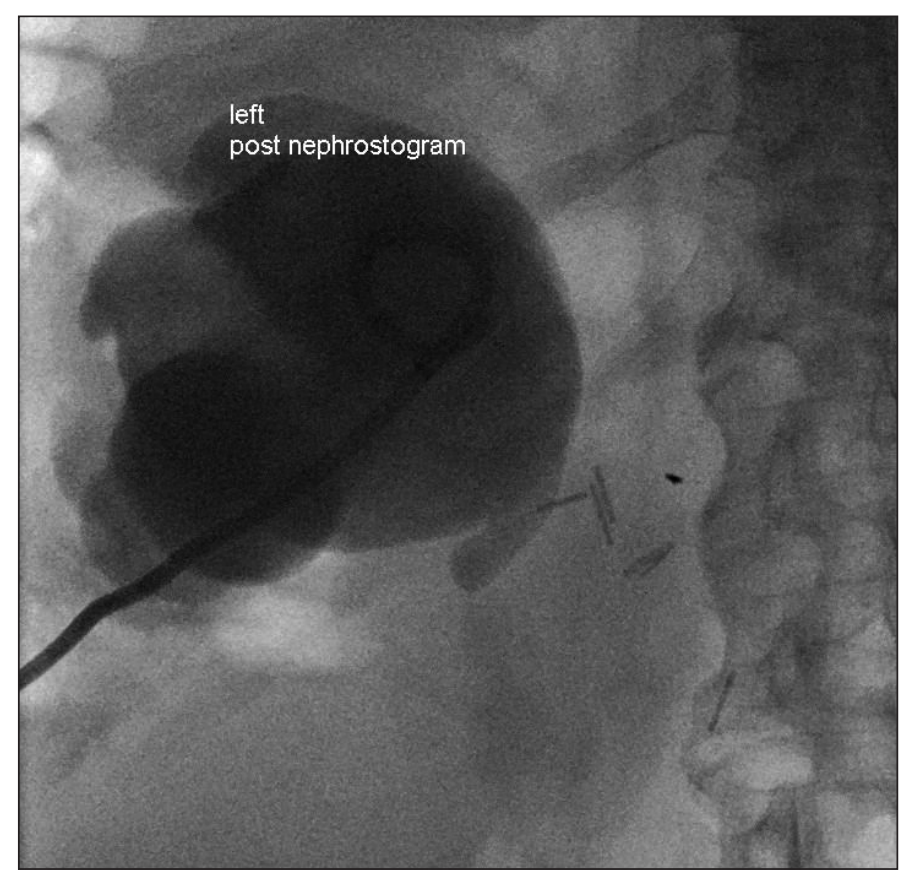

Fig. 3. Anterograde nephrostogram showing contrast extravasation and hydronephrosis.

placed for 4 to 6 weeks. At the site of the reconstruction, a retroperitoneal Penrose drain should be placed to help limit urinoma formation. This drain should be maintained for 48 hours, or until any urinary extravasation subsides. The bladder should be decompressed with a Foley catheter for 2 to 7 days. A suprapubic catheter may be used if necessary. ${ }^{5}$ Serial follow-up pyelography at 3 and 6 months should be carried out to ensure a lack of ureteral anastamotic complications. $^{5}$

In delayed diagnosis of urinoma, percutaneous catheter drainage of the fluid can be utilized. If a grade I or grade II ureteral injury is present, careful stenting to ensure drainage to the bladder may be carried out. ${ }^{5}$ Renal function should be

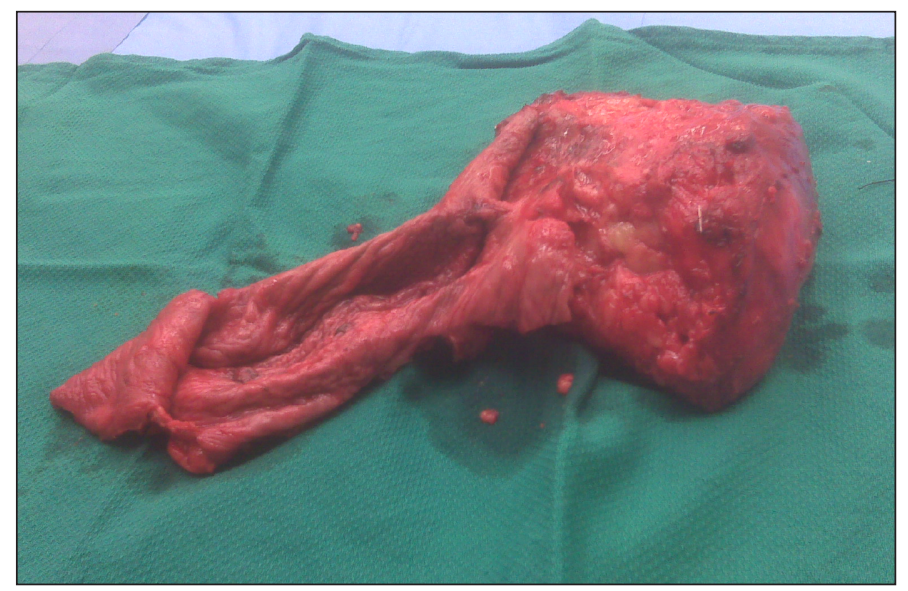

Fig. 4. Urinoma mass after excision via open procedure. The mass contained urinoma, involved left ureter, kidney and adrenal.

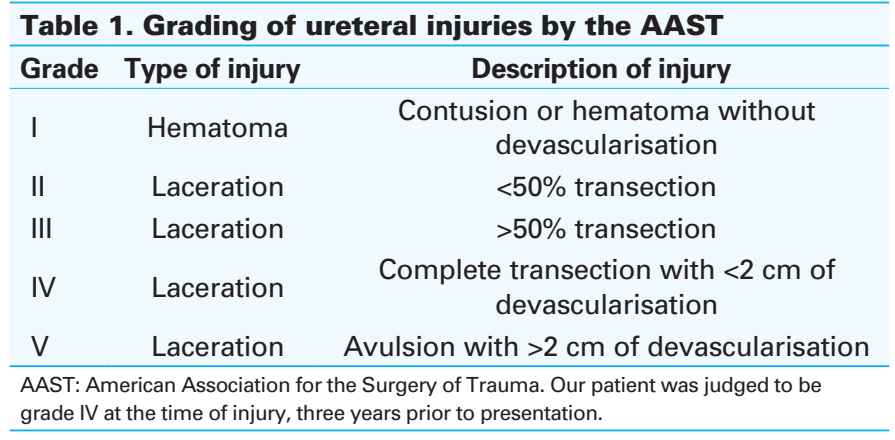

closely monitored. Contrast CT or ultrasound should be used to ensure resolution. ${ }^{7}$ In low-grade injuries, these actions alone may be therapeutic. ${ }^{5}$ More intensive surgical drainage and reconstruction tend to be required in high-grade injuries, recurrent urinoma and hemodynamic instability. ${ }^{7}$ If surgical reconstruction is to be attempted, this should be delayed until the acute periureteral inflammatory response is resolved, if possible. ${ }^{5}$ Selective renal artery embolization or nephrectomy may be used in rare instances. ${ }^{7}$

Prevention of ureteric injury during aortic surgery is important. The core principles for prevention are an excellent understanding of ureteric anatomy, intra-operative recognition of the ureter and meticulous surgical technique during dissection adjacent to the ureter. Preoperative ureteric localization studies and ureteric stenting have been used, but have not been shown to reduce the risk of ureteric injury. They should not substitute for adherence to the previously stated core principles. ${ }^{1}$

\section{Conclusion}

Ureteric injury is a rare complication of abdominal aortic aneurysm repair. While extrinsic compression is the most common type of injury, direct injury may also occur. Ureteric injuries in this setting frequently have a delay in diagnosis, and a high mortality. It is important to maintain an increased level of suspicion in patients who have had surgery adjacent to the ureter. Options for diagnostic imaging include retrograde pyelogram or CT scan. Delayed images should be performed if a fluid collection is identified. Treatment is guided by grade of injury and whether identification is acute or delayed. Core principles to prevent ureteric injury during aortic surgery have been described. Adherence to these principles is important to reduce this rare, but serious, complication.

Competing interests: None declared.

This paper has been peer-reviewed. 
Large minimally symptomatic iatrogenic urinoma

\section{References}

1. York JW, Money SR. Prevention and management of ureteral injuries during aortic surgery. Semin Vasc Surg 2001;145:266-74. http://dx.doi.org/10.1053/svas.2001.27887

2. Sheehan MK, Shireman PK, Littooy FN, et al. Ureteral injury during aortic aneurysm repair by the retroperitoneal approach. Ann Vasc Surg 2001;155:481-4. http://dx.doi.org/10.1007/s100160010125

3. Guttikonda S, Vitellas K. Ureteral leak after abdominal aortic aneurysm repair: case report. Emerg Radiol 2002;92:103-5.

4. Elliot SP, McAninch JW. Ureteral injuries: external and iatrogenic. Urol Clin North Am 2006;331:55-66. http://dx.doi.org/10.1016/i.ucl.2005.11.005

5. Armenakas NA. Current method of diagnosis and management of ureteral injuries. World I Urol 1999;172:78-83. http://dx.doi.org/10.1007/s003450050110
6. Dalsing MC, Bihrle R, Lalka SG, et al. Vascular surgery-associated ureteral injury: zebras do exist. Ann Vasc Surg 1993;72:180-5. http://dx.doi.org/10.1007/BF02001013

7. Cheng KS. Urinoma: a rare complication of abdominal aortic aneurysm surgery. ScientificWorldJournal 2004;4(S1):59-61. http://dx.doi.org/10.1100/tsw.2004.48

8. Moore EE, Cogbill TH, Malangoni M, et al. Injury scoring scale. 1992. http://www.aast.org/Library/ TraumaTools/InjuryScoringScales.aspx. Accessed March 12, 2012.

Correspondence: Mark Spurrell, Memorial University of Newfoundland, 20 Edmonton Place, St. John's, NL AIA 2N7; fax: 709-753-6605; rmspurre@mun.ca 